\title{
Evaluation of antimicrobial metabolites produced by Arthrobacter kerguelensis VL-RK_09 by GC-MS analysis
}

\author{
Rajesh Kumar Munaganti ${ }^{1}$, Vijayalakshmi Muvva ${ }^{{ }^{*}}$, Mustafa Oskay², Mani Deepa Indupalli ${ }^{1}$ \\ ${ }^{1}$ Department of Botany and Microbiology, Acharya Nagarjuna University, Guntur-522510, A.P, India, +91 \\ 0863234 6339, profmuvva@gmail.com \\ 2 Section of Microbiology, Department of Biology, Faculty of Sciences and Letters, Celal Bayar University, \\ Manisa, Turkey, +90 236201 3265, mustafa.oskay@cbu.edu.tr \\ ${ }^{*}$ Corresponding author / İletişimden sorumlu yazar

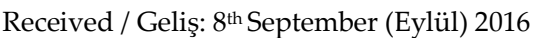 \\ Accepted / Kabul: 14 $4^{\text {th }}$ November (Kasim) 2016 \\ DOI: $10.18466 /$ cbayarfbe.280596
}

\begin{abstract}
The purpose of the present study was concerned with the isolation and characterization of a rare actinobacterial strain designated as VL-RK_09 from a Mango orchard and evaluation of its antimicrobial compounds by GC-MS analysis. Soil dilution plate technique was employed for the isolation of the strain on yeast extract malt extract dextrose (YMD) agar medium. The strain was identified as Arthrobacter kerguelensis based on polyphasic taxonomy. Suitable culture media for the production of antimicrobial metabolites was assessed by inoculating the strain in different ISP (International Streptomyces project) media and non ISP media. The culture broth of the strain grown in best suitable medium (ISP-2) was extracted with different solvents viz., chloroform, ethyl acetate, methanol and acetone. The culture broth inoculated in ISP-2 and extracted with ethyl acetate exhibited strong antimicrobial activity against oppurtunistic pathogenic microorganisms tested. The crude ethyl acetate extract exhibiting high antimicrobial activity was analysed by Gas Chromatography-Mass Spectroscopy to reveal the metabolites produced by the strain and evidenced the presence of 39 compounds according to the available library data, NIST MS Search (ver. 2.0). The results of the present study revealed the production of diversified metabolites by the strain and hence this strain could be a possible source for novel antimicrobial compounds.

Keywords - Arthrobacter kerguelensis, antimicrobial metabolites, gas chromatography, International Streptomyces Project, mass spectroscopy, polyphasic taxonomy.
\end{abstract}

\section{Introduction}

Natural bioactive compounds remain the best source of drugs and drug leads that serve as outstanding small molecule probes to dissect fundamental biological reactions. The number of new chemical entities of microbial origin that has been approved by the Food and Drug Administration (FDA) has been reduced in the past decade. This scarcity can be partly attributed to the redundancy in the discovered molecules from microbial isolates, which are isolated from common terrestrial ecolog- ical units. A great challenge for the natural product community is to discover novel natural products efficiently and cost effectively [1-4].

The development of resistance in microbes is the leading cause of short-lived euphoria over the discovery of a new drug in addition to the drug's toxicity. Hence, there is always a compelling need for discovering new drugs with newer mechanisms to tackle this menace. Advances in basic research have enabled scientists to understand the course of disease and the way a drug works at the molecular level. New families of anti-infective compounds are needed to enter the market place at regular inter- 
vals to tackle the new diseases caused by evolving pathogens. Emerging infectious organisms often encounter hosts with no prior exposure to them and thus represent a novel challenge to the host's immune system. Several viruses responsible for human epidemics have made a transition from animal host to humans and are now transmitted from human to human.

Microbes continue to play a highly considerable role in the drug discovery and development process. Nevertheless, however, this situation can be partly overcome by exploring rarely exploited ecological niches as the source of microbes, which reduces the chances of isolating compounds similar to existing ones. The use of modern and advanced isolation techniques, modification of the existing fermentation methods, genetic modifications to induce expression of silent genes, analytical tools for the detection and identification of new chemical entities, use of polymers in fermentation to enhance yield of fermented compounds, and so on, have all aided in enhancing the frequency of acquiring novel compounds. These compounds are representative of numerous classes of diverse compounds. Thus, compounds of microbial origin and their analogues undergoing clinical trials continue to demonstrate the importance of compounds from microbial sources in modern drug discovery.

The need to access novel bacterial species is an urgent practical issue, as biotech and pharmaceutical industries appear to culture and screen microbial taxa that seem to be repeatedly re-isolating. In our search for isolation and identification of novel species and exploration for novel bioactive compounds, we have isolated A. kerguelensis VL-RK_09, a rare actinobacterial strain with potential antimicrobial activity. In the present study an attempt was made to evaluate the antimicrobial compounds by GC-MS analysis.

\section{Materials and Methods}

\subsection{Chemicals}

All the solvents and reagents used in this experiment were of extra pure grade procured from Merck (Mumbai, India).

\subsection{Strain Isolation and Identification}

The potent rare actinobacterial strain A. kerguelensis VL-RK_09 was isolated from the soil samples collected at a Mango orchard of Vissannapet, Andhrapradesh, India by soil dilution plate technique on YMD agar medium composed of malt extract $(1 \%)$, yeast extract $(0.4 \%)$, dextrose $(0.4 \%), \mathrm{CaCO}_{3}$ $(0.2 \%)$ and agar $2.0 \%(\mathrm{pH} 7.0 \pm 0.2)$. The strain was identified by polyphasic approach and the $16 \mathrm{~S}$ rRNA gene sequence of the strain has been deposited in NCBI Genbank with an accession number KJ787652. ISP-2 grown culture (4 days of post inoculation) was used to observe the micromorphology of the strain through scanning electron microscopy (SEM) according to the method described by Bozzola and Russell [13]. The strain was maintained on YMD agar medium at $4^{\circ} \mathrm{C}$ for further studies [14].

\subsection{Screening and Selection of Suitable Culture Media for Bioactive Metabolite Production}

The antagonistic activity of the strain A. kerguelensis VL-RK_09 was evaluated by growing in different ISP (International Streptomyces Project) and Non ISP media such as ISP-1, ISP-2, ISP-3, ISP-4, ISP-5, ISP-6, ISP-7 and starch casein salts broth. During the fermentation process, growth as well as yield of bioactive metabolites by the strain was recorded. The medium which favours high yield of bioactive metabolite production by the strain was fixed for further studies. The antimicrobial activity of the strain was determined by agar well diffusion assay. The homogenous culture suspension prepared by suspending three day old culture in sterile saline was used to inoculate different media and incubated at $30^{\circ} \mathrm{C}$ for 5 days on a rotator shaker at 180 rpm. Antimicrobial compound was recovered from the filtrate by solvent extraction method and the residue thus obtained was used to determine antimicrobial activity.

\subsection{Fermentation and Extraction of Antimicrobial Metabolites}

The antimicrobial efficacy of the A. kerguelensis VLRK_09 was evaluated by extracting the fermentation broth with four solvents such as methanol, acetone, chloroform and ethyl acetate. Among the solvents used, ethyl acetate extract exhibited maximum antimicrobial activity where as the other solvent extracts showed moderate to minimum 
CBÜ Fen Bil. Dergi., Cilt 12, Sayı 3, 355-361 s

activity against the test microorganisms.

For extraction and characterization of antimicrobial metabolites, actively growing pure culture of the strain was inoculated into the production medium $(20 \mathrm{~L})$ composed of $0.4 \%$ dextrose, $0.4 \%$ yeast extract, $1 \%$ malt extract, $0.2 \%$ calcium carbonate $(\mathrm{pH}$ 7.2). The flask was incubated on a rotary shaker $(180 \mathrm{rpm})$ at $30^{\circ} \mathrm{C}$ for 5 days. The fermentation was stopped after 5 days and the culture broth obtained after filtration was extracted twice with ethyl acetate and concentrated under reduced pressure to yield a crude extract $(2 \mathrm{~g})$.

\subsection{Characterization of Antimicrobial Metabolites by GC-MS Analysis}

The components of the crude ethyl acetate extract of the strain A. kerguelensis VL-RK_09 was analysed on Agilent GC-MS system (GC: 5890 series II; MSD 5972). The fused-silica HP-5 capillary column (30 $\mathrm{m} \times 0.25 \mathrm{~mm}$, ID, film thickness of $0.25 \mu \mathrm{m}$ ) was directly coupled to the MS. The carrier gas was helium with a flow rate of $1.2 \mathrm{~mL} / \mathrm{min}$. Oven temperature was programmed $\left(50^{\circ} \mathrm{C} / \mathrm{min}\right.$, then 50 $280^{\circ} \mathrm{C}$ at rate of $5^{\circ} \mathrm{C} / \mathrm{min}$ ) and, subsequently, held isothermally for $20 \mathrm{~min}$. The temperature of the injector port was maintained at $250^{\circ} \mathrm{C}$ and that of detector at $280^{\circ} \mathrm{C}$. The peaks of components in gas chromatography were subjected to mass spectral analysis. The spectra were analysed from the available library data, NIST MS Search (ver. 2.0) (included with NIST'02 mass spectral library, Agilent p/n G1033A).

\subsection{Test Organisms}

The antimicrobial activity of strain was determined against several opportunistic pathogenic bacteria and fungi.

Bacteria: Staphylococcus aureus MTCC 3160, Bacillus megaterium NCIM 2187, Shigella flexneri MTCC 1457, Lactobacillus casei MTCC 1423, L.acidophilus MTCC 495, Xanthomonas campestris MTCC 2286, Proteus vulgaris MTCC 7299, Pseudomonas aeruginosa ATCC 9027, Escherichia coli ATCC 35218, Salmonella typhi, Vibrio cholera and Streptococcus mutans MTCC 497.

Fungi: Candida albicans ATCC 10231, Aspergillus niger, A. flavus, Fusarium solani, F. oxysporum MTCC
CBU J. of Sci., Volume 12, Issue 3,p 355-361

3075, Penicillium citrinum and Alternaria sp.

\subsection{Biological Assay}

The antimicrobial activity of metabolites produced by the strain A. kerguelensis VL-RK_09 was determined by agar well diffusion method [15]. Nutrient agar (NA) and Czapek-Dox (CD) agar media were used for culturing the test bacteria and fungi respectively. NA medium $(100 \mathrm{~mL})$ was sterilized at $15 \mathrm{lbs}$ pressure $\left(121^{\circ} \mathrm{C}\right)$ for $15 \mathrm{~min}$, cooled and inoculated with $0.1 \mathrm{~mL}$ of test bacterial suspension. After thorough mixing, the inoculated medium was poured into Petri plates under aseptic conditions. After solidification of agar medium, wells of about $6 \mathrm{~mm}$ diameter were punched with sterilized cork borer. In case of antifungal assay, test fungus $\left(10^{5}\right.$ spores $/ \mathrm{mL}$ ) was plated onto the solidified CD agar plate. Ethyl acetate extract (50 ppm) was added to each well while the addition of only ethyl acetate served as control. The inoculated plates were incubated at $30^{\circ} \mathrm{C}$ and the diameter of the inhibition zone was measured after $24 \mathrm{~h}$ of incubation for bacteria and 24-72 $\mathrm{h}$ for yeast and filamentous fungi.

\section{Results and Discussion}

The potent rare actinobacterial strain isolated from Mango orchards of Vissannapet, which exhibited strong antagonistic activity, was identified as $A$. kerguelensis VL-RK_09. The detailed micromorphological studies through scanning electron microscopy revealed that the cells of the strain displayed rudimentary branching in the exponential phase and fragmented into coryneforms and coccoid elements at the stationary phase exhibiting the rod-coccus life style $[16,17]$. The SEM photograph of the strain is presented in Figure 1. 


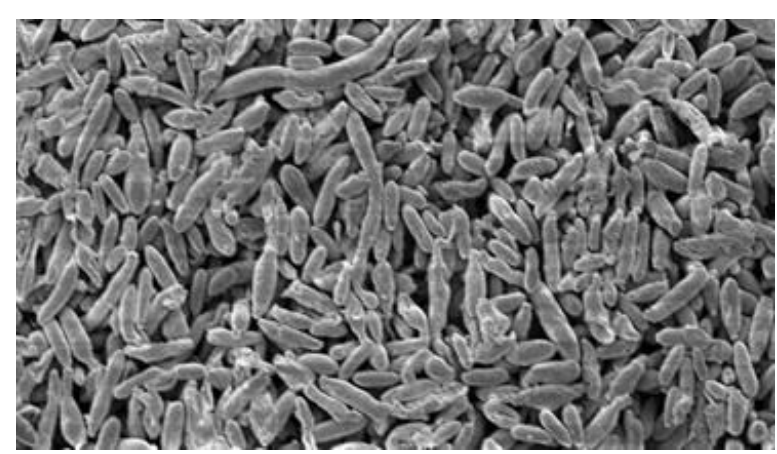

Figure 1. SEM image showing the pleomorphic Arthrobacter cells.

\subsection{Selection of Suitable Culture Media for Bioactive Metabolite Production}

Among the different ISP and non ISP media tested, ISP-2 medium supported high yields of antimicrobial metabolites by the strain followed by peptone yeast extract iron agar medium (ISP-6) and inorganic salts starch agar (ISP-4) (Figure 2). The metabolites produced by the strain showed very little effect on the test organisms when cultured in ISP-1. Modified yeast extract malt extract dextrose broth (ISP-2) favoured maximum production of antimicrobial metabolites by Pseudonocardia sp. UK-10 and Rhodococcus erythropolis VL-RK_09 isolated from mangrove soils and Mango orchards respectively $[18,19]$.

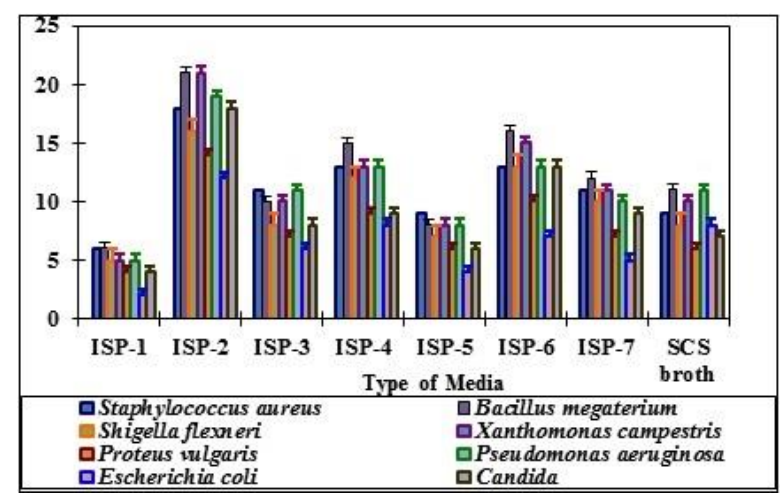

Figure 2. Influence of different media on the production of antimicrobial metabolites by A. kerguelensis VL-RK_09.

Similarly Naragani et al., [20] reported that yeast extract malt extract dextrose agar supported the maximum bioactive metabolite production by a marine isolate Rhodococcus erythropolis VLK-12. Among the different solvents tested, ethyl acetate found to be the suitable solvent, where as the other solvent extracts showed moderate to minimum activity against the test microorganisms (Table 1).

Table 1. Antimicrobial activity of different solvent extracts of A. kerguelensis VL-RK_09.

\begin{tabular}{lcccc} 
& \multicolumn{4}{c}{ Diameter of zone of inhibition (mm) } \\
\hline Test organisms & $\mathrm{A}^{*}$ & $\mathrm{~B}$ & $\mathrm{C}$ & $\mathrm{D}$ \\
\hline S. aureus & 14 & 18 & 16 & 13 \\
B. megaterium & 16 & 22 & 20 & 14 \\
S. flexneri & 15 & 18 & 16 & 13 \\
X. campestris & 17 & 21 & 18 & 15 \\
P. vulgaris & 12 & 16 & 14 & 13 \\
P. aeruginosa & 15 & 18 & 17 & 14 \\
E. coli & 12 & 15 & 13 & 11 \\
C. albicans & 13 & 18 & 15 & 12 \\
\hline
\end{tabular}

${ }^{*} \mathrm{~A}$-Chloroform extract; B - Ethyl acetate extract; C - Methanol extract; D - Acetone extract

\subsection{Extraction and Characterization of Antimi- crobial Metabolites by GC-MS Analysis}

The fermentation was stopped after 5 days and the culture broth obtained after filtration was extracted twice with ethyl acetate and concentrated under reduced pressure to yield a dark brown crude extract $(2 \mathrm{~g})$. Analysis of the components of ethylacetate extract by GC revealed the presence of 39 peaks at different retention times (Figure 3).

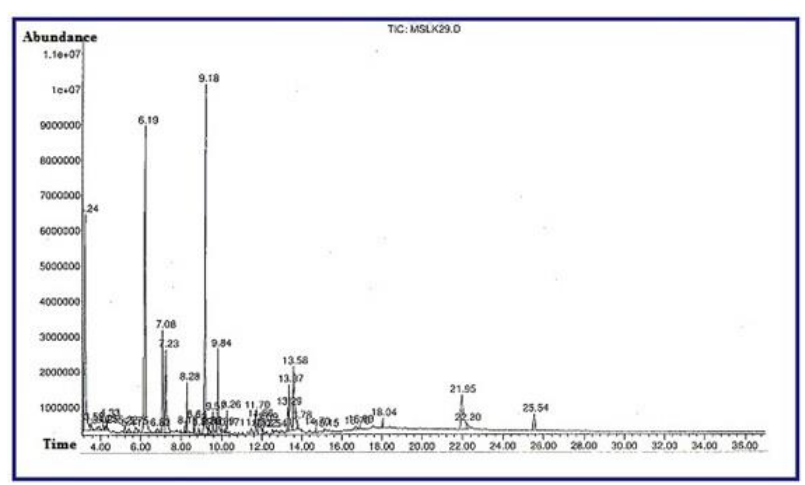

Figure 3. GC MSD spectrum of ethyl acetate extract of A. kerguelensis VL-RK_09.

According to the available library data, NIST MS Search (ver. 2.0) (included with NIST '02 mass spectral library, Agilent p/n G1033 A), compounds viz., 1 to 39 present crude ethyl acetate extract were tentatively identified and the details of the compounds are presented in Table 2 
Table 2. Antimicrobial compounds identified in ethyl acetate extract by GC-MS.

\begin{tabular}{|c|c|c|c|}
\hline $\begin{array}{c}\text { Peak } \\
\text { No }\end{array}$ & Compound Name & $\begin{array}{l}\text { Retention } \\
\text { Time }\end{array}$ & Area $\%$ \\
\hline 1 & Benzene acetic acid & 3.24 & 14.48 \\
\hline 2 & 17-Pentatriacontene & 3.53 & 0.83 \\
\hline 3 & Butanoic acid & 3.95 & 0.54 \\
\hline 4 & 3-Octanone & 4.15 & 0.41 \\
\hline 5 & 1-Dodecene & 4.25 & 0.2 \\
\hline 6 & Tetradecene & 4.25 & 0.41 \\
\hline 7 & alpha-Farnasene & 5.22 & 0.41 \\
\hline 8 & $\begin{array}{l}\text { 7H-1,3-Dioxolo }[4,5] \text { furo }[3,2- \\
\text { d] }[1,3] \text { dioxin }\end{array}$ & 5.41 & 0.49 \\
\hline 9 & $\begin{array}{l}\text { 4-Amino-2,6- } \\
\text { dihydroxypyrimidine }\end{array}$ & 5.75 & 0.73 \\
\hline 10 & beta Phenylethyl butyrate & 6.19 & 19.11 \\
\hline 11 & N-Propyl-butyramide & 6.83 & 0.53 \\
\hline 12 & Propanoic acid & 7.08 & 5.29 \\
\hline 13 & 2-Pentanone & 7.23 & 6.42 \\
\hline 14 & 3-Eicosene & 8.18 & 0.19 \\
\hline 15 & Octadecene & 8.28 & 1.5 \\
\hline 16 & 1,2-Benzenedicarboxylic acid & 8.64 & 0.55 \\
\hline 17 & 10-Nonadecanone & 8.89 & 0.36 \\
\hline 18 & Mefenoxam & 9.18 & 15.92 \\
\hline 19 & Pentadecanoic acid & 9.39 & 0.4 \\
\hline 20 & Dibutyl Pthalate & 9.57 & 1.08 \\
\hline 21 & n-Hexadecanoic acid & 9.83 & 4.64 \\
\hline 22 & Z-8-Hexadecene & 10.17 & 0.23 \\
\hline 23 & Heptadecene & 10.26 & 0.63 \\
\hline 24 & 13-Octadecenol & 11.47 & 0.68 \\
\hline 25 & Octadecanoic acid & 11.7 & 1.33 \\
\hline 26 & 1,1-Biphenyl-3-amine & 11.86 & 1.59 \\
\hline 27 & 1-Heptacosanol & 12.02 & 0.23 \\
\hline 28 & Docosane & 12.09 & 0.41 \\
\hline 29 & 9,10-Anthracenedione & 12.54 & 0.32 \\
\hline 30 & 1H-1,2,4-Triazole & 13.29 & 1.24 \\
\hline 31 & 2-Thiophenecarboxylic acid & 13.37 & 2.29 \\
\hline 32 & [1,1-Biphenyl]-4-amine & 13.57 & 5.64 \\
\hline 33 & Nonadecane & 13.78 & 0.44 \\
\hline 34 & Carbazole & 15.15 & 0.22 \\
\hline 35 & E-Z-8,10-Dodecadien-1-ol & 16.7 & 0.48 \\
\hline 36 & 3,7,11-Tridecatrienenitrile & 16.89 & 0.15 \\
\hline 37 & $\begin{array}{l}\text { 3-[4-Methoxyphenyl] quino- } \\
\text { lin-4-ol }\end{array}$ & 18.04 & 0.39 \\
\hline 38 & Phthalic anhydride & 21.94 & 5.27 \\
\hline 39 & Quinoline & 22.2 & 0.79 \\
\hline
\end{tabular}

The ethyl acetate extract of A. kerguelensis VLRK_09 exhibited good antimicrobial activity against Gram positive, Gram negative bacteria and fungi (Table 3). Among the bacteria tested, Bacillus megaterium was highly sensitive to the metabolites followed by Pseudomonas aeruginosa, Staphylococcus aureus, Xanthomonas campestris, Streptococcus mutans, Shigella flexneri, Corynebacterium diptheriae, Lactobacillus casei, L. acidophilus, Proteus vulgaris, Vibrio cholerae and Salmonella typhi. Among the fungi tested, Candida albicans exhibited high sensitivity followed by Aspergillus niger, A. flavus, Fusarium solani, F.oxysporum, Penicillium citrinum, and Alternaria sp.

Table 3. Antimicrobial activity of crude ethyl acetate extract of A. kerguelensis VL-RK_09.

\begin{tabular}{ll}
\hline Test Microorganisms & Zone of inhibition $\mathbf{( m m )}$ \\
\hline Bacteria & \\
\hline Staphylococcus aureus & 34 \\
Bacillus megaterium & 37 \\
Shigella flexneri & 28 \\
Xanthomonas campestris & 32 \\
Proteus vulgaris & 26 \\
Pseudomonas aeruginosa & 33 \\
Escherichia coli & 24 \\
Salmonella typhi & 18 \\
Streptococcus mutans & 25 \\
Vibrio cholera & 24 \\
Lactobacillus casei & 26 \\
L. acidophilus & 25 \\
\hline Fungi & \\
\hline Candida albicans & 29 \\
Aspergillus niger & 25 \\
A. flavus & 22 \\
Fusarium solani & 21 \\
F. oxysporum & 20 \\
Penicillium citrinum & 18 \\
Alternaria sp. & 21 \\
\hline Values are mean \pm S.E.M (n = 3) &
\end{tabular}

Designing the appropriate fermentation medium is a crucial step in the production of secondary metabolites [21]. Prior knowledge and experience in developing a suitable basal medium may play an important role in further medium optimization [22]. Secondary metabolite production is often influenced by various environmental factors including nutrients (nitrogen and carbon source), oxygen supply, temperature, $\mathrm{pH}$ [23-25]. The genus Arthrobacter has not been well explored for the production of sec- 
ondary metabolites though there were a few reports. Arthrobacter sp. isolated from three different Antarctic sponges produced antimicrobial compounds active against Burkholderia cepacia complex. The antimicrobial compounds belong to the class of volatile organic compounds (VOCs). A novel exopolysaccharide, B4EPS produced by Arthrobacter sp. B4 was reported to inhibit biofilm formation by Pseudomonas aeruginosa PAO1 [26].

Actinobacteria are a physiologically diverse group and are of great importance in biotechnological processes because of their ability to produce a large number of antibiotics, enzymes, and other therapeutically useful secondary metabolites with diverse biological activities with original and unforeseen structures, and are selective inhibitors of their molecular targets.

\section{Conclusion}

In the present study, the strain Arthrobacter kerguelensis VL-RK_09 exhibited strong antimicrobial activity against oppurtunistic pathogenic bacteria and fungi when cultured in ISP-2 under controlled conditions. The analysis of crude ethyl acetate extract by GC-MS analysis revealed the presence of important antimicrobial compounds and hence the strain could be used as a source of novel antimicrobial compounds.

\section{Acknowledgements}

The author R.K thanks UGC for providing the financial assistance to carry out the research work and the Department of Botany and Microbiology for providing the laboratory facilities.

\section{References}

[1] Baltz, R.H. Antibiotic discovery from actinomycetes: will a renaissance follow the decline and fall? SIM News 2005; 55, 186-196.

[2] Baltz, R.H. Antimicrobials from actinomycetes: back to the future. Microbe 2007; 2, 125-131.

[3] Bull, A.T.; Ward, A.C.; Goodfellow, M. Search and discovery strategies for biotechnology: the paradigm shift. Microbiol. Mol. Biol. Rev. 2000; 64, 573-606.

[4] Valan Arasu, M.; Duraipandiyan, V.; Agastian, P.; Ignacimuthu, S. Antimicrobial activity of Streptomy- ces spp. ERI-26 recovered from Western Ghats of Tamil Nadu. J. Med. Mycol. 2008; 18, 147-153.

[5] Euzéby, J.P. List of bacterial names with standing in nomenclature: a folder available on the Internet. Int. J. Sys. Bacteriol. 1997; 47, 590-592.

[6] Kamigiri, K. YM-30059, a novel quinolone antibiotic produced by Arthrobacter sp. J. Antibiot. 1996; 49, 823-825.

[7] Hentschel, U. Isolation and phylogenetic analysis of bacteria with antimicrobial activities from the Mediterranean sponges Aplysina aerophoba and Aplysina cavernicola. FEMS Microbiol. Ecol. 2001; 35, 305312.

[8] Li, J.Q.; Tan, B.P.; Mai, K.S.; Ai, Q.H.; Zhang, W.B.; Xu， W.; Liufu, Z.G.; Ma, H.M. Comparative study between probiotic bacterium Arthrobacter XE-7 and chloramphenicol on protection of Penaeus chinensis post-larvae from pathogenic vibrios. Aquaculture 2006; 253, 140-147.

[9] Lo Giudice, A.; Brun, V.; Michaud, L. Characterization of Antarctic psychrotrophic bacteria with antibacterial activities against terrestrial microorganisms. J. Basic Microbiol. 2007; 47, 496-505.

[10] Salonius, K.; Siderakis, C.; MacKinnon, A.M.; Griffiths, S.G. Use of Arthrobacter davidanieli as a live vaccine against Renibacterium salmoninarum and Piscirickettsia salmonis in salmonids. Progr. Fish Vaccinol. 2005; 121, 189-197.

[11] Wiese, J.; Thiel, V.; Nagel, K.; Staufenberger, T.; Imhoff, J.F. Diversity of antibiotic-active bacteria associated with the brown alga Laminaria saccharina from the Baltic Sea. Marin. Biotechnol. 2009; 11, 287300.

[12] Rojas, J.L. Bacterial diversity from benthic mats of Antarctic lakes as a source of new bioactive metabolites. Marin. Genom. 2009; 2, 33-41.

[13] Bozzola, J.J.; Russell, L.D. Specimen Preparation for Transmission Electron Microscopy. Electron Microscopy: Principles and Techniques for Biologists. Sudbury, Mass.: Jones and Bartlett. 1999; pp. 21-31.

[14] Shirling, E.B.; Gottlieb, D. Methods for characterization of Streptomyces sp. Int. J. Sys. Bacteriol. 1966; $16,313-340$.

[15] Cappuccino, J.G.; Sherman, N. Microbiology, a laboratory manual. Pearson Education, Inc., New Delhi, 2004; pp. 282-283.

[16] Germida, J.J.; Casida, L.E. Myceloid growth of Arthrobacter globiformis and some other Arthrobacter species. J. Bacteriol. 1980; 144, 1152-1158.

[17] Mohamed Ahmed, I.A.; Arima, J.; Ichiyanagi, T.; Sakuno, E.; Mori, N. Isolation and characterization of 
3-N-trimethylamino-1-propanol degrading Arthrobacter sp. strain E5. Res. J. Microbiol. 2009; 4, 49-58.

[18] Usha Kiranmayi, M.; Sudhakar, P.; Sreenivasulu, K.; Vijayalakshmi, M. Optimization of Culturing Conditions for Improved Production of Bioactive Metabolites by Pseudonocardia sp. VUK-10. Mycobiol. 2011; 39, 174-181.

[19] Munaganti, R.K.; Muvva, V.L.; Naragani, K.; Bindhu B.S.S.N. Cultural parameters influencing the production of Antimicrobial Metabolites by Rhodococcus erythropolis VL-RK_05. Int. J. Curr. Res. 2015; 7, 14924-14931.

[20] Naragani, K.; Munaganti, R.K.; Mangamuri, U.K.; Muvva V.L. Optimization of Culture Conditions for Enhanced Antimicrobial Activity of Rhodococcus erythropolis VLK-12 Isolated from South Coast of Andhra Pradesh, India. Brit. Microbiol. Res. J. 2014; 4, 59-75.

[21] Gao, H.; Liu, M.; Liu, J.; Dai, H.; Zhou, X.; Liu, X.; Zhuo, Y.; Zhang, W.; Zhang, L. Medium optimization for the production of avermectin B1a by Streptomyces avermitili 14-12A using response surface methodology. Biores. Technol. 2009; 100, 4012-4016.

[22] Jia, B.; Jin, Z.; Mei, L. Medium Optimization Based on Statistical Methodologies for Pristinamycins Production by Streptomyces pristinaespiralis. Appl. Biochem. Biotechnol. 2008; 144, 133-143.

[23] Lin, J.; Bai, L.; Deng, Z.; Zhong, J. Effect of Ammonium in Medium on Ansamitocin P-3 Production by Actinosynnema pretiosum. Biotechnol. Biopro. Engin. 2010; 15, 119-125.

[24] Ruiz, B.; Chavez, A.; Forero, A.; Garcia-Huante, Y.; Romero, A.; Sanchez, M.; Rocha, D.; Sanchez, B.; Rodruguez-Sanoja, R; Sanchez, S.; Langley, E. Production of microbial secondary metabolites: Regulation by the carbon source. Crit. Rev. Microbiol. 2010; 36, 146-167.

[25] Sánchez, S.; Chavez, A.; Forero, A.; GarciaHuante, Y.; Romero, A.; Sánchez, M.; Rocha, D.; Sánchez, B.; Avalos, M.; Guzman-Trampe, S.; Rodriguez Sanoja, R.; Langley, E.; Ruiz, B. Carbon source regulation of antibiotic production. J. Antibiot. 2010; 63, 442-459.

[26] Li, Y.; Li, Q.; Hao, D.; Jiang, D.; Luo, Y.; Liu, Y.; Zhao, Z. Production, Purification, and Antibiofilm Activity of a Novel Exopolysaccharide from Arthrobacter sp. B4. Prep. Biochem. Biotechnol. 2015; 45, 192-204 
CBÜ Fen Bil. Dergi., Cilt 12, Sayı 3, 355-361 s

CBU J. of Sci., Volume 12, Issue 3,p 355-361

XVZ 